\title{
Development of Spaceborne Antenna for Circularly Polarized SAR Using Kevlar Honeycomb Core
}

\author{
Yuto Osanai \\ Center for Environmental Remote Sensing, Chiba University, Chiba 263-8522, Japan
}

\begin{abstract}
This paper presents the development of circularly polarized microstrip antenna using Kevlar honeycomb core as the substrates for application of CP-SAR (circularly polarized synthetic aperture radar) which signal propagation is not affected by Faraday rotation effect in the ionosphere, as compared to linearly polarized systems especially at the L-band. The reason why Kevlar honeycomb core is used is because it is suitable for space environment in terms of lightweight and resistant to heat and shock. Measurements of the fabricated antenna were performed to confirm the simulation results. The results show good characteristics of the antennas except the axial ratio. The reason of insufficient axial ratio is assumed as fabrication error of the radiator and microstrip line.
\end{abstract}

Key words: Kevlar honeycomb core, CP-SAR, spaceborne antenna, microstrip antenna.

\section{Introduction}

It is essential to monitor natural disasters such as earthquake, volcanic eruption, flood and landslide to protect human life. Remote sensing from space is appropriate as a method of natural disasters monitoring. The remote sensing for natural disasters can be useful in three steps: before, while and after natural disaster happens. One example of remote sensing for natural disaster before the natural disaster happens is making hazard map. Hazard map enables us to predict how serious the natural disaster will be when it happens and helps people to prevent from it. Remote sensing also will be useful while natural disaster happens because it can provide people whole image of a place where natural disaster happens. That whole image helps people to think how to evacuate and where to go to help first. After natural disaster happens, remote sensing also can give hint which will be so useful when recovering plan is considered.

There are mainly two types of sensor for remote sensing from space: optical sensor and microwave

Corresponding author: Yuto Osanai, Master, research field: Antenna engineering. sensor. Because microwave sensor can get data regardless weather and condition of day and night, it is suitable for monitoring of the natural disaster that is impossible to predict when to occur. A microwave sensor onboard microsatellite is SAR (synthetic aperture radar).

SAR is one type of radar. The SAR system is mounted on a moving platform such as airplane or microsatellite. It can realize a bigger aperture by moving a long distance on the platform and induce high resolution image. Standard radar is called real aperture radar. But real aperture radar can only achieve low resolution image. SAR can obtain higher resolution data compared to real aperture radar thanks to its synthetic aperture technique by moving its platform.

JMRSL (Josaphat Microwave Remote Sensing Laboratory) is developing L-band CP-SAR (circularly polarized synthetic aperture radar) onboard a microwave satellite. L-band microwave is adequate to natural disaster monitoring because it can penetrate leaves and branches of trees. There are two advantages of circular polarization. The first advantage of circular polarization is that circular polarization antenna can get both of vertical and 
horizontal polarization not like linear polarization antenna. Linear polarization antenna just can get a polarization which direction is same as the direction of the antenna. The second advantage to use circular polarization is that it can calculate Faraday rotation effect [1]. Faraday rotation effect is an effect which changes polarization plane. If linear polarization is used as a polarization of SAR onboard microsatellite, the polarization plane will change and it causes unprecise information of earth surface. If circular polarization is used instead of linear polarization, Faraday rotation effect can be compensated because the angle of change in the ionosphere can be calculated from some formula using electric density. Circular polarization is more convenient technique in case of using L-band because Faraday rotation effects in the ionosphere get stronger using longer wavelength. In other words, L-band CPSAR is a so expected solution for natural disaster monitoring.

UAV (unmanned aerial vehicle) can be used as a platform for CP-SAR to check its function. UAV was also developed in JMRSL [2]. Circularly polarized array antenna for the CPSAR sensor was developed to mount on the UAV [3]. A base-band signal processor using FPGA and onboard PC was also developed for test using the UAV in JMRSL [4].

An antenna is needed for L-band CPSAR onboard microsatellite. The antenna has to have enough characteristic at three points which are light weight and resistant to heat and shock to stand harsh space environment. A material which satisfies these three points is Kevlar honeycomb core.

Kevlar is the registered trademark for para-aramid synthetic fiber, related to other aramids such as Nomex and Technora. It was developed by Stephanie Kwolek at DuPont in 1965 [5]. This high-strength material was first commercially used in early 1970s as replacement for steel in racing tires, and is still used in various applications today.

Honeycomb is a mass of hexagonal wax cells built by honeybees in their nests to contain their larvae and stores of honey and pollen. In this paper, the word "honeycomb" is used just to mean the shape of the substrate. Fig. 1 shows an image of the Kevlar honeycomb core which is shaped similar to a bee's honeycomb. Some researchers have already studied characteristics of the honeycomb structure [6-8]. As honeycomb structure is suitable for space environment, slot antenna with honeycomb structures for space application has developed [9]. X-Band Parallel-Plate Slot Array Antenna for SAR Sensor onboard $100 \mathrm{~kg}$ Small Satellite was also developed using the honeycomb core [10]. Additionally, the honeycomb material is used for Dual Polarized UHF/VHF Stacked-Patch Feed Array for a Large-Aperture Space-borne Radar Antenna [11]. Table I shows strength characteristic of the Kevlar honeycomb core.

A goal of this research is to know characteristic of the microstrip antenna using Kevlar honeycomb core to realize the L-band CPSAR onboard the microstate which is under development in JMRSL. To archive this goal, microstrip single patch antenna is using Kevlar honeycomb core. This paper presents simulation and measured results of RHCP (right-handed circularly polarized) and LHCP (left-handed circularly polarized) microstrip antennas utilizing Kevlar honeycomb core substrate. Based on this research, the number of antenna patches on the substrates planned to be added to form an array, and then it will realize the required specifications for the CP-SAR.

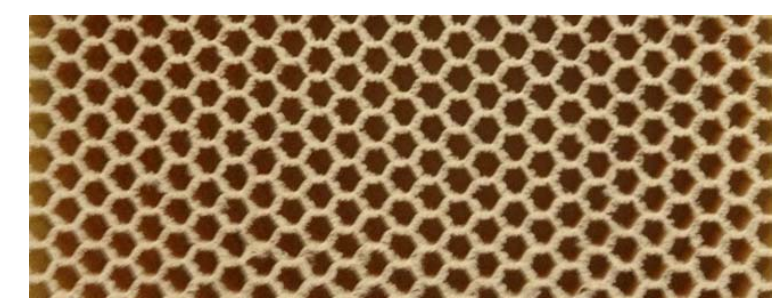

Fig. 1 Image of Kevlar honeycomb core.

Table 1 Characteristic of Kevlar honeycomb core.

\begin{tabular}{llll}
\hline \multicolumn{2}{c}{ L Direction } & \multicolumn{2}{c}{ W Direction } \\
\hline Strength(psi) & Modulus(ksi) & Strength(psi) & Modulus(ksi) \\
\hline 130 & 18 & 69 & 9.3 \\
\hline
\end{tabular}




\section{Antenna Design}

Table 2 shows specifications for CP-SAR antenna onboard microsatellite. The research has been done to satisfy these specifications in the future. To achieve these antenna characteristics, a single patch microstrip antenna was designed, fabricated, and measured as a first step. Therefore the targeted parameters to satisfy in this specification are bandwidth, axial ratio, and $\mathrm{S}_{11}$. The other parameters, such as beamwidth and gain, will be satisfied by developing array antenna in the future.

Figs. 2 and 3 show the fabricated RHCP and LHCP antennas. The shape of the antenna's radiating patch is square which corner is truncated to make the circularly polarized radiation beam.

As shown in Figs. 2 and 3, the Kevlar honeycomb substrate has many holes. These holes can cause disagreement between simulation and measurement because the holes cannot be realized in simulation software. The dielectric constant and loss tangent of Kevlar honeycomb core are measured by using a Dielectric Probe Kit shown in Fig. 4. The probe to touch the Kevlar honeycomb core is connected to the network analyzer and data gotten from the network analyzer is sent to PC in Fig. 4. The corner and center point are chosen as points to put the probe on. The measurement using the dielectric probe kit has done three times. The result is that dielectric constant is 1.137, which is almost same as air, and loss tangent is 0.065 .

As shown in Fig. 5, the bottom layer of the antenna is copper ground. The thickness of the honeycomb substrate is $6 \mathrm{~mm}$. Fig. 6 shows size of both RHCP

Table 2 Specification of the antenna.

\begin{tabular}{ll}
\hline Parameter & Specification \\
\hline Bandwidth & $<5$ degrees \\
Beamwidth & $>20 \mathrm{MHz}$ \\
Axial ratio & $<3 \mathrm{~dB}$ \\
Gain & $>15 \mathrm{dBic}$ \\
$\mathrm{S}_{11}$ & $<-10 \mathrm{~dB}$ \\
Center-Frequency & $1.275 \mathrm{GHz}$ (L-band) \\
\hline
\end{tabular}

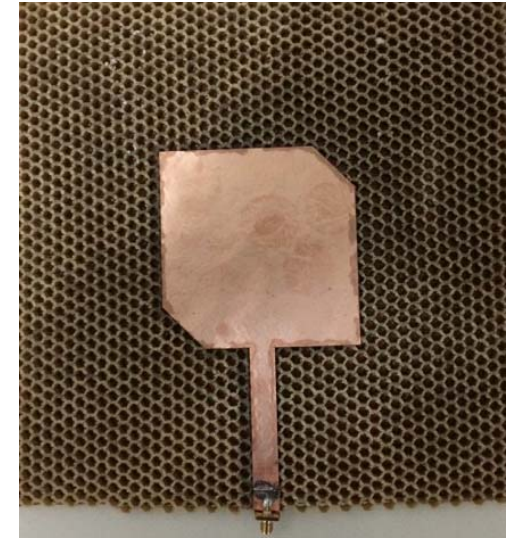

Fig. 2 Right handed circularly polarized antenna.

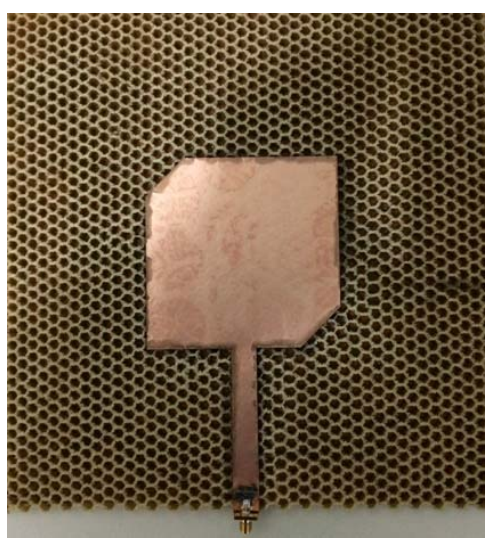

Fig. 3 Left handed circularly polarized antenna.

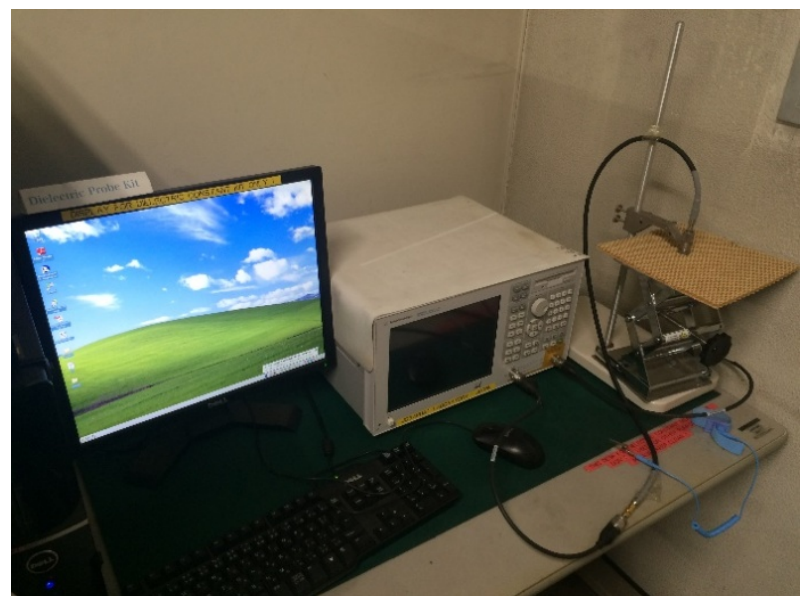

Fig. 4 Dielectric probe kit.

and LHCP antenna. The size of antenna that includes substrate is $267 \mathrm{~mm}$ by $267 \mathrm{~mm}$. The size of the radiating patch is $101 \mathrm{~mm}$ by $101 \mathrm{~mm}$ before the corner is truncated.

The fabricated antenna was measured in the anechoic chamber of JMRSL, CEReS (Center of Environmental Remote Sensing), Chiba University. Fig. 7 shows the 


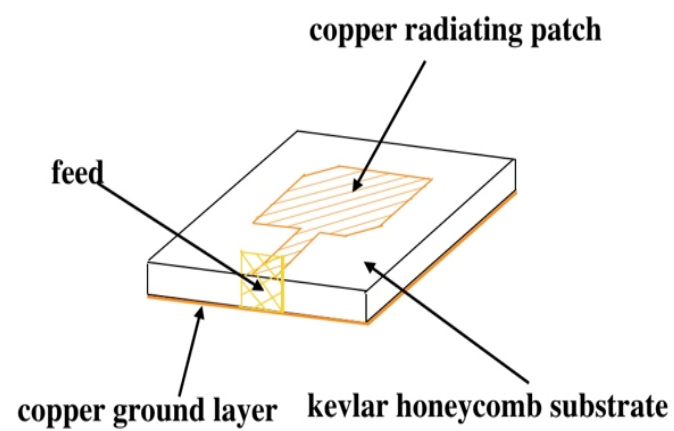

Fig. 5 Side view of the antenna.

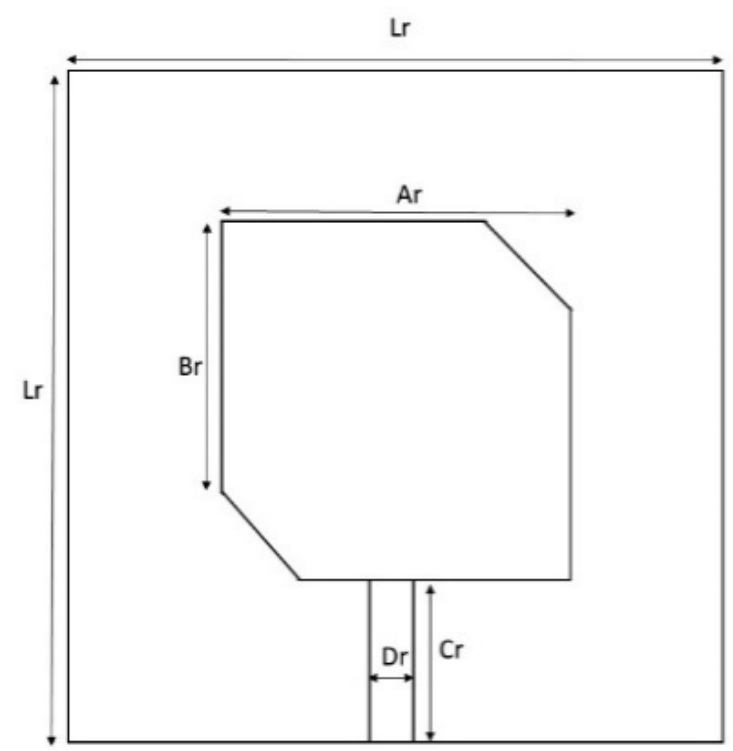

(a)RHCP

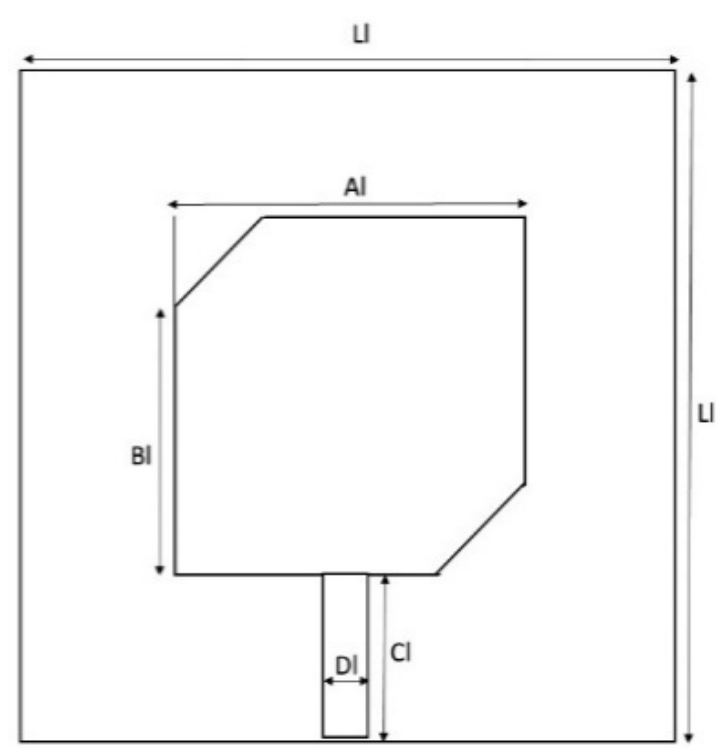

(b) LHCP

\begin{tabular}{|l|l|l|l|l|l|l|l|l|l|l|}
\hline Parameter & $\mathrm{Ar}$ & $\mathrm{Br}$ & $\mathrm{Cr}$ & $\mathrm{Dr}$ & $\mathrm{Lr}$ & $\mathrm{Al}$ & $\mathrm{Bl}$ & $\mathrm{Cl}$ & $\mathrm{Dl}$ & Ll \\
\hline Value $(\mathrm{mm})$ & 101 & 79.8 & 83 & 13 & 267 & 101 & 79.8 & 83 & 13 & 267 \\
\hline
\end{tabular}

Fig. 6 Size of each antenna.

radiation pattern measurement set up. RHCP and LHCP conical long spiral antenna are used as the transmitting antenna in the anechoic chamber. The conical long spiral antenna and the antenna under test are connected to a VNA (vector network analyzer). The black sharp objects in Fig. 7 are RF absorbers.

AR (axial ratio) of AUT (antenna under test) can be calculated using RHCP and LHCP transmitting antenna. The equation can be given as Eq. (1).

$$
\mathrm{AR}(\mathrm{dB})=20 \log _{10}\left|\frac{P_{R}+P_{L}}{P_{R}-P_{L}}\right|
$$

where, $P_{R}$ means receiving power from RHCP corn antenna and $P_{L}$ means receiving power from LHCP corn antenna. Gain can be calculated by using Eq. (2).

$$
\text { Gain }(\mathrm{dBic})=P_{A U T}(\mathrm{~dB})-P_{\text {dipole }}(\mathrm{dB})+2.15-3
$$

All of values in Eq. (2) are dB unit. $P_{A U T}$ is power that the microstrip antenna using Kevlar honeycomb core receives. A dipole antenna is also measured to induce gain value. Receive power by the dipole antenna is expressed as $P_{\text {dipole }}(\mathrm{dB}) .2 .15$ needs to be added to change the unit to $\mathrm{dBi}$ from $\mathrm{dBd}$. As the polarization is circular, polarization loss factor 3 also needs to be added to get circular polarization gain. 


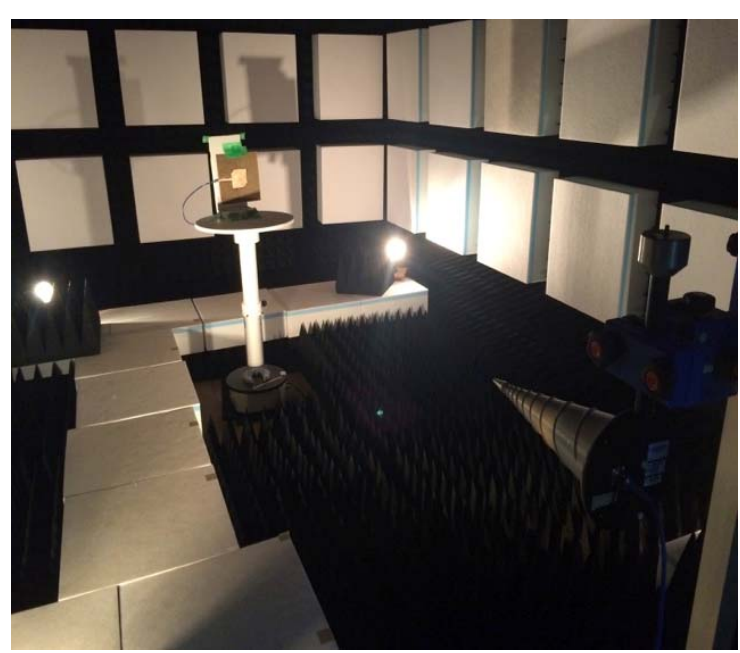

Fig. 7 Measurement set up.

\section{Results and Discussion}

Figs. 8-12 show simulation and measured results for $\mathrm{S}_{11}$, axial ratio, and radiation pattern of RHCP and LHCP antennas. Table 3 shows the summary of results.

Fig. 8 shows measurement results for both RHCP and LHCP antennas. The value of $S_{11}$ at the point of center frequency of $1.275 \mathrm{GHz}$ is approximately -20 $\mathrm{dB}$ while the simulation result is approximately -40 $\mathrm{dB}$. There is disagreement between simulation and measurement result for both RHCP and LHCP at the minimum value of $S_{11}$. Even though there are approximately $20 \mathrm{~dB}$ difference between simulation and measurement result of $S_{11}$, the measurement value is enough to work as an antenna because the value of $\mathrm{S}_{11}$ at the center frequency of $1.275 \mathrm{GHz}$ is below -10 $\mathrm{dB}$. The shape of graph has good agreement between simulation and measurement graph for $\mathrm{S}_{11}$ even though the depth is $20 \mathrm{~dB}$ different. Fig. 8 also shows results of bandwidth. Measurement result of bandwidth for both of RHCP and LHCP antennas is approximately $90 \mathrm{MHz}$ while the simulation result is approximately $180 \mathrm{MHz}$. Therefore the difference between simulation and measurement of bandwidth is approximately $90 \mathrm{MHz}$. Although there is $90 \mathrm{MHz}$ bandwidth difference between simulation and measurement result, the results succeed to satisfy the specification in Table 2.

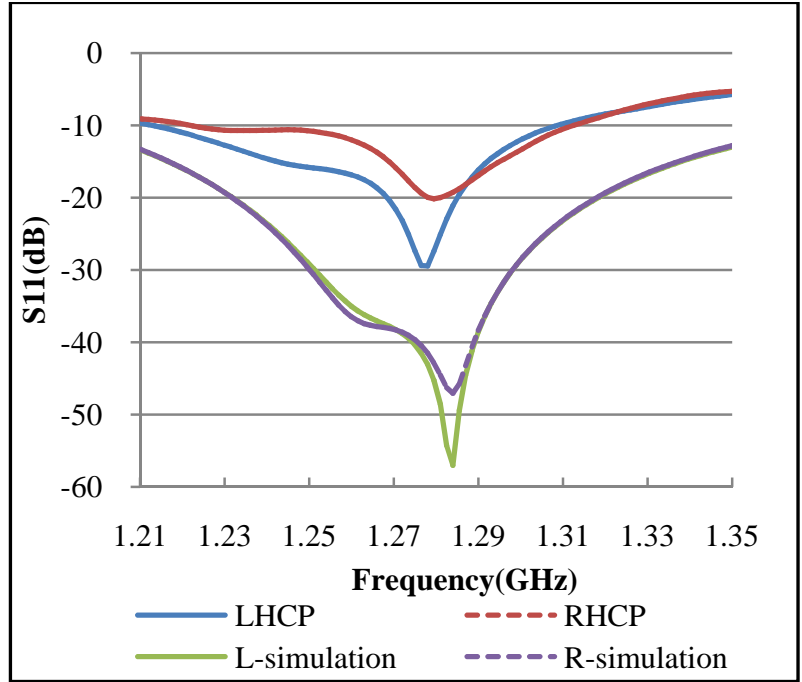

Fig. 8 Simulated and measured results of S11.

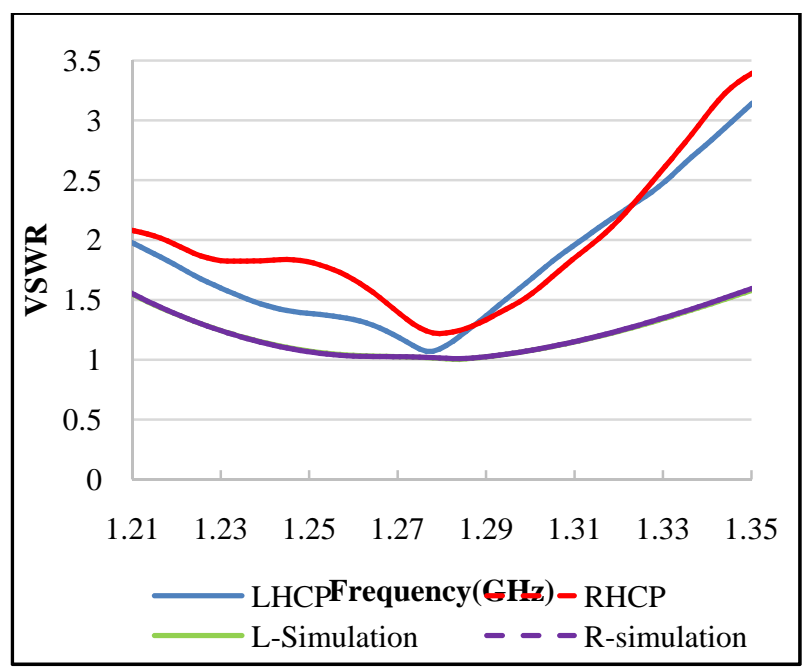

Fig. 9 Simulated and measured results of VSWR.

The results of VSWR (voltage standing wave ratio) are shown in Fig. 9. The results show simulation and measurement results for both RHCP and LHCP. A term in the graph "R-simulation" means simulation result of RHCP antenna. In the same way, a term "L-simulation" means simulation result of LHCP antenna. Furthermore a term "LHCP" in the figure means measurement result of LHCP antenna and a term "RHCP" means measurement result of RHCP antenna. Each result has same center frequency value as the results of $S_{11}$. The simulation results of VSWR for RHCP and LHCP are 1.01 and 1.00 respectively as significant figures. The measurement results of VSWR for RHCP and LHCP are 1.22 and 1.07 
respectively as significant figures. Typically, it is said that value of VSWR less than 2 is enough for antenna. Therefore, the values which could take from this result satisfy the requirement.

Fig. 10 shows simulation and measurement results of axial ratio in decibel for RHCP and LHCP antennas. As shown in Fig. 10, the simulation value at the point of center frequency $1.275 \mathrm{GHz}$, has approximately 0 $\mathrm{dB}$ for both of RHCP and LHCP antennas, which mean the transmission beam can be circularly polarization. But the measurement result for both of RHCP and LHCP antennas shows that the value at the point of center frequency $1.275 \mathrm{GHz}$ does not reach to below $3 \mathrm{~dB}$, which means the polarization is not sufficiently circular, even though the corner of radiator is truncated to radiate circularly polarization.

The reason why the value of axial ratio is insufficient is considered from fabrication error. Three main problems were found from the axial ratio difference between simulation and measurement. The first problem is that simulation results did not include effect of glue that is for attachment between the copper and the honeycomb substrate. The second problem is losses from connector. The third problem is heterogeneity of dielectric constant of the substrate that occurred by the substrate's holes. The solution for these problems is to put the Kevlar honeycomb substrate between two high strength dielectric substrates such as carbon composite. The third problem might be figured out by estimating the value of dielectric constant for simulation from the difference between these results of simulation and measurement.

Fig. 11 shows simulation and measurement radiation pattern for LHCP antenna. Radiation pattern of E-plane and H-plane is described for both of simulation and measurement results. The radiation pattern of E-pane and H-pane has good agreement because it is considered that symmetric radiation patch radiate symmetric radiation beam. The results of simulation and measurement also have good agreement. The radiation pattern is typical one of the microstrip single

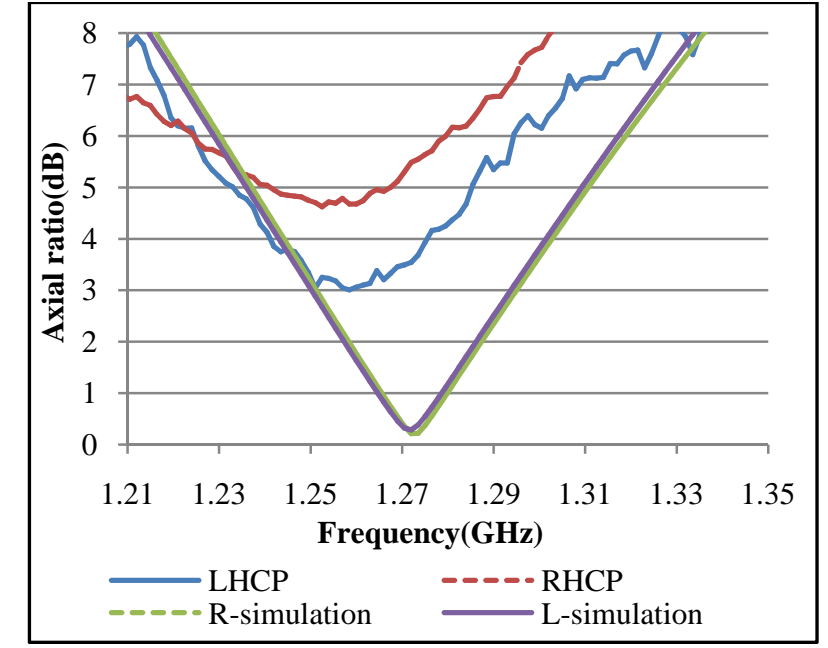

Fig. 10 Simulated and measured results of axial ratio.

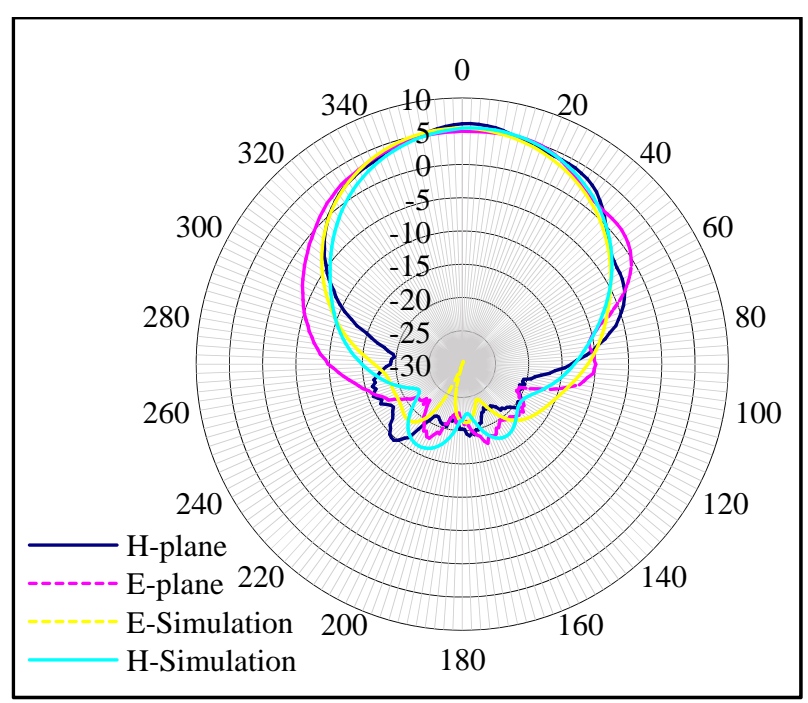

Fig. 11 Left handed circularly polarized radiation pattern @ 1.275 GHz.

patch antenna although dielectric substrate is Kevlar honeycomb core. Fig. 11 does not only show radiation pattern but also value of gain. As shown in Fig. 10 and Table 3, the gain value is $5-6 \mathrm{dBi}$. The beamwidth of the fabricated antenna can also read from Fig. 10. HPBW (half power beam width) for both E-plane and H-plane can be seen in Fig. 10 and Table 3. The HPBW of E-plane is approximately 10 degrees bigger than the HPBW of H-plane. Fig. 12 shows simulation and measurement radiation patterns for the RHCP antenna. The results have similar characteristics with LHCP antennas radiation pattern, gain and HPBW. 


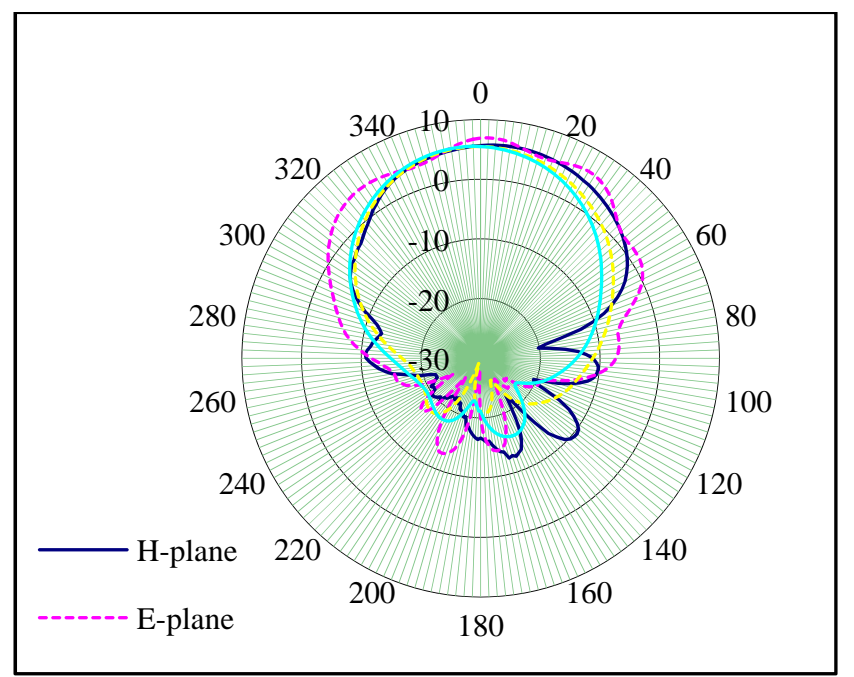

Fig. 12 Right handed circularly polarized radiation pattern @ 1.275 GHz.

Table 3 Measured and simulated antenna parameters.

\begin{tabular}{|c|c|c|c|c|c|c|c|c|}
\hline & \multicolumn{2}{|c|}{ RHCP-Measure } & \multicolumn{2}{|c|}{ RHCP-Simulation } & \multicolumn{2}{|c|}{ LHCP-Measure } & \multicolumn{2}{|c|}{ LHCP-Simulation } \\
\hline Band-Width & \multicolumn{2}{|c|}{$90 \mathrm{MHz}$} & \multicolumn{2}{|c|}{$180 \mathrm{MHz}$} & \multicolumn{2}{|c|}{$92 \mathrm{MHz}$} & \multicolumn{2}{|c|}{$183 \mathrm{MHz}$} \\
\hline \multirow{2}{*}{ Beam-Width } & H-plane & E-plane & H-plane & E-plane & H-plane & E-plane & H-plane & E-plane \\
\hline & 70 deg & $84 \mathrm{deg}$ & $56 \operatorname{deg}$ & $60 \mathrm{deg}$ & 60 deg & $70 \mathrm{deg}$ & 58 deg & 60 deg \\
\hline Axial Ratio & $5.64 \mathrm{~dB}$ & & $0.37 \mathrm{~dB}$ & & $3.93 \mathrm{~dB}$ & & $0.54 \mathrm{~dB}$ & \\
\hline Gain & $5.62 \mathrm{dBi}$ & & $5.49 \mathrm{dBi}$ & & $6.13 \mathrm{dBi}$ & & $5.49 \mathrm{dBi}$ & \\
\hline S11 & $-18.60 \mathrm{~dB}$ & & $-39.64 \mathrm{~dB}$ & & $-27.37 \mathrm{~dB}$ & & $-39.64 \mathrm{~dB}$ & \\
\hline Center-Frequency & $1.275 \mathrm{GHz}$ & L-band) & & & & & & \\
\hline
\end{tabular}

Table 3 is a summary of the results. The values of the Table 3 come from Figs. 8-12. The most substantial difference between simulation and measurement is occurred in axial ratio.

\section{Conclusion}

This paper presents development of two microstrip antennas (LHCP and RHCP) for CP-SAR using Kevlar honeycomb core as the substrate. Kevlar honeycomb core is used as the substrate because it is lightweight and resistant to shock and heat, therefore it is appropriate for space application. The fabricated antenna was measured in an anechoic chmber to verify the simulation results. The results show that both of RHCP and LHCP antennas are working at the center frequency of $1.275 \mathrm{GHz}$. The radiation pattern of the Kevlar honeycomb core single patch antenna is similar in shape to the typical patch antenna radiation pattern. The disagreement between simulation and measurement of axial ratio is considered to happen due to fabrication error in microstrip line and radiator. The presented research will be continued by adding patches to make array antenna to satisfy the final specification of the CP-SAR antenna.

\section{Acknowledgment}

JMRSL (Josaphat Microwave Remote Sensing Laboratory) would like to thank the Japanese Ministry of Education and Technology (Monbukagakusho), for supporting JMRSL.

\section{References}

[1] Sumantyo, J. T. S., Jae-Hyun, K., and Kim, T. H. 2014. "Progress on Development of Synthetic Aperture Radar onboard UAV and Microsatellite.” Presented at the 2014 International Conference on Information and Communication Technology Convergence (ICTC), Busan, Korea.

[2] Sumantyo, J. T. S., Chet, K. V., and Triharjanto, R. H. 2013. "Development of Circularly Polarized Synthetic 
Aperture Radar Onboard Unmanned Aerial Vehicle.” In Proceedings of 2013 IEEE International Geoscience and Remote Sensing Symposium, 2301-4.

[3] Wissan, V., Firmansyah, I., Rizki Akbar, P., Sri Sumantyo, J. T., and Kuze, H. 2011. "Development of Circularly Polarized Array Antenna for Synthetic Aperture Radar Sensor Installed on UAV.” Progress in Electromagnetics Research C (19): 119-33.

[4] Suto, K., Sri Sumantyo, J. T., Koo, V. C., Chua, M. Y., and Cheaw, W. G. 2014. "Development of SAR Base-Band Signal Processor Using FPGA and Onboard PC.” In Proceedings of 2014 IEEE International Geoscience and Remote Sensing Symposium, 672-5.

[5] Mera, H., and Takata, T. 2002. "High-Performance Fibers.” In Ullmann's Encyclopedia of Industrial Chemistry.Wiley-VCH, Weinheim.

[6] Nakano, H., Tajima, S., Suzuki, N., Mimaki, H., and Yamauchi, J. 1997. "The Radiation Characteristics of Honeycomb Antennas.” In Proceedings of Antennas and Propagation, Tenth International Conference 1 (436): 350-3.

[7] Yang, L., and Zhang, X. S. 2010. "Simulation on Long-Wave Radiation Restraining Performance of Honeycomb Structure." Presented at the 2010 Asia-Pacific Power and Energy Engineering Conference,
China.

[8] Zhou, P., Huang, L., Xie, J., Liang, D., Lu, H., and Deng, L. 2012. "A Study on the Effective Permittivity of Carbon/PI Honeycomb Composites for Radar Absorbing Design.” IEEE Transactions on Antennas and Propagation 60 (8): 3679-83.

[9] Nguyen, T., Sakurai, K., Hirokawa, J., Ando, M., Amano, O., Koreeda, S., and Kamata, Y. 2014. "A Concise Design of Large mm-Wave Radial Line Slot Antenna with Honeycomb Structures for Space Application.” In Proccedings of 2014 XXXIth General Assembly and Scientific Symposium, 1-4.

[10] Akbar, P. R., Saito, H., Zang, M., Hirokawa, J., and Ando, M. 2015. "X-band Parallel-Plate Slot Array Antenna for SAR Sensor Onboard $100 \mathrm{~kg}$ Small Satellite.” In Proceedings of 2015 IEEE International Symposium o $n$ Antennas and Propagation \& USNC/URSI National Radio Science Meeting, 208-9.

[11] Partridge, P., Moghaddam, M., Rahmat-Samii, Y., Haynes, M., Van Nieuwstadt, L., Vitaz, J., and Cable, V. 2008. “A Dual Polarized UHF/VHF Honeycomb Stacked-Patch Array Antenna: Overview of an Enabling Technology for the MOSS Mission.” In Proceedings of Antennas and Propagation Society International Symposium (AP-S 2008), 1-4. 